\title{
Atypical mycobacterial infections following laparoscopic surgery
}

\author{
WAMP Samaranayake ${ }^{1}$, KMMP Dassanayake $^{1}$ \\ Sri Lankan Journal of Infectious Diseases 2018 Vol.8 (1):32-35 \\ DOI: http://dx.doi.org/10.4038/sljid.v8i1.8160
}

\begin{abstract}
Three cases of atypical mycobacterial infection were encountered following laparoscopic surgery at the Colombo North Teaching Hospital over a period of eighteen months from September 2014 to April 2016. The patients presented with port site infections and sinus tract formation three to four weeks after laparoscopic surgery performed in the same operating theatre. These cases were clinically suspected as atypical mycobacterial infection. The Ziehl Neelsen stain showed acid fast bacilli with atypical morphology and cultures grew mycobacteria other than tuberculosis (MOTT). Histology of tissues showed chronic granulomatous inflammation. The infections resolved with surgical intervention and long term antibiotic therapy. Erroneous practice of disinfection of laparoscopic instruments with formaldehyde was thought to be the cause of these infections. No cases were reported after implementing protocols for proper cleaning and disinfection of laparoscopes.
\end{abstract}

Keywords: Laparoscopic surgery, Atypical mycobacterial infection, Disinfection

\section{Introduction}

Laparoscopic procedures have become the practice of choice for many surgical conditions. Atypical mycobacterial infection at port sites is an increasing problem encountered in patients undergoing laparoscopic surgery and has been reported to cause nosocomial outbreaks. ${ }^{1,2,3,4}$ It leads to increased morbidity and long term treatment. Multidrug resistance further complicates the problem. ${ }^{1}$ Atypical mycobacteria have been known to present in water and soil and can therefore easily contaminate solutions, instruments and disinfectants used in hospital settings. Erroneous cleaning, disinfection and sterilization of laparoscopic instruments is almost always responsible for such outbreaks. ${ }^{2}$

\section{Materials and methods}

We encountered three patients with atypical mycobacterial infection after laparoscopic interventions performed at the same operating theatre in Colombo North Teaching Hospital from September 2014 to April 2016. The patients presented with post laparoscopic non healing persistent discharging sinuses. At the time of discharge, none of them had showed any signs of surgical wound infections. None of the patients complained of a febrile illness.

${ }^{1}$ Colombo North Teaching Hospital, Ragama, Sri Lanka

Address for correspondence: Dr WAMP Samaranayake. No 57/17, Railway Station Road, Veyangoda, Sri Lanka. Telephone: +94772955484 Email: manorisamaranayake1981@gmail.com

(iD https://orcid.org/0000-0001-5279-9166.

Received 3 November 2017 and revised version accepted 24 April 2018 
Case 1

A 46 year old diabetic patient presented with port site infection three weeks after laparoscopic adhesiolysis. Local examination showed a sinus discharging seropurulent fluid at the port site with accompanying induration and pigmentation around the site. Gram staining and fungal staining did not show any organisms and culture of pus did not grow any organisms. Acid fast stain of pus showed acid fast bacilli with atypical morphology. GeneXpert-TB PCR was negative. Culture yielded mycobacteria other than tuberculosis (MOTT) at the National Tuberculosis Reference Laboratory (NTRL), Welisara, Sri Lanka. Sinus tract explorations and wound debridement with a three month course of cotrimoxazole, amikacin and doxycycline resolved the infection.

Case 2

A 36 year old female presented with laparoscopic port site infection and sinus tract formation four weeks after laparoscopic ligation and resection of fallopian tubes. Gram staining and fungal staining did not show any organisms and culture did not grow any organisms. Histology of tissue revealed suppurative granulomatous inflammation with multi nucleated giant cells. Acid fast stain showed acid fast bacilli with atypical morphology. GeneXpert-TB PCR was negative. Culture carried out at NTRL yielded MOTT. Sinus tract removal with extensive debridement along with a three month course of ciprofloxacin and co-trimoxazole resolved the infection.

Case 3

A 40 year old male presented with painful, erythematous swellings at the port site three weeks after laparoscopic inguinal hernia repair. USS abdomen showed pus collection in the peritoneum which was drained surgically. His C-reactive protein was high. Peritoneal pus yielded a multi-drug resistant coliform and acid fast stain showed acid fast bacilli with atypical morphology. Mycobacterium spp. was not isolated on culture. The mesh was explored and removed partially and the patient responded to a course of intravenous meropenem for 7 days. Patient was discharged and followed up at the clinic for one year.

On investigation, we found that laparoscopic instruments were cleaned inadequately in between surgical procedures, disinfection was done by immersion in a solution using a formalin tablet and kept overnight, and the storage of the instruments was done inside a sealed box without proper ventilation. Several measures were taken to rectify the situation which included education of theatre staff regarding proper cleaning, reduction of number of surgical procedures per day, thereby facilitating adequate time in between for cleaning, cessation of the practice of using formalin tablets for disinfection, use of peracetic-acid as high level disinfection, provision of adequate amount of autoclaved water for rinsing, proper storage of laparoscopic instruments, and plan to purchase a plasma sterilizer in the future.

\section{Discussion}

Atypical mycobacterial infections following laparoscopic surgery differ from those caused by other bacteria in terms of clinical manifestations, diagnosis and management. ${ }^{1}$ Atypical mycobacteria species (e.g. Mycobacterium chelonae, $M$. fortuitum) are widely distributed in 
soil and water, and can therefore contaminate hospital instruments. ${ }^{2,3,4}$ The three patients described above presented with discharging sinuses in and around the port site of indolent onset without systemic manifestations 3-4 weeks after surgery.

The presence of acid fast bacilli in the Ziehl-Neelsen stain of pus followed by positive culture of atypical mycobacteria in the first 2 patients helped make the diagnosis. Clinical suspicion and acid fast stain is important for early diagnosis as isolation of mycobacteria from pus or tissues takes a long time. The treatment of choice for these infections is surgical debridement along with prolonged antimicrobial therapy. ${ }^{1,4,5,6}$ Proper antibiotic treatment for atypical mycobacterial infections is a matter of debate. Combinations of second line anti-tuberculosis drugs are preferred due to limited response to first-line drugs. Most of the regimens consist of combinations of quinolones, macrolides, and aminoglycosides. The duration of treatment ranges from 6 weeks to 4 months. ${ }^{1,2}$ Two patients were given recommended combination of antibiotics together with wound debridement. Wide local excision of discharging sinuses was performed in all three cases.

Prevention of these infections is challenging as mycobacteria are resistant to killing by disinfection due to their high lipid cell wall content and their existence within a biofilm. ${ }^{1}$ In the three patients, the assumption was that acquisition of atypical mycobacteria occurred at surgery from contaminated laparoscopes.

The use of disposable laparoscopic instruments is the gold standard but this is not practicable in Sri Lanka due to cost constraints. Reprocessing of laparoscopic instruments includes proper cleaning, disinfection/sterilization, rinsing, drying and storage. Presoaking of instruments prevents drying of blood and removes blood from the instruments. Thorough cleaning manually or mechanically (e.g. ultrasonic cleaners or washer decontaminators/disinfectors) is required before high-level disinfection/ sterilization because inorganic and organic materials that remain on the surfaces of instruments interfere with the effectiveness of these processes. ${ }^{7}$ A neutral or near-neutral $\mathrm{pH}$ detergent solution with or without enzymes is commonly used for cleaning due to its material compatibility profile and good soil removal effect. ${ }^{7}$ The Centre for Disease Control and Prevention recommends that laparoscopes and other scopes that enter normally sterile tissue should be sterilized (steam sterilization, ethylene oxide or plasma sterilization) before each use or should receive at least high-level disinfection (glutaraldehyde, stabilised hydrogen peroxide and per acetic acid) although the latter practice continues to be debated. ${ }^{7}$ Rinsing with sterile water followed by drying with alcohol or forced air and storage in a way that prevents recontamination and promotes drying would further safeguard quality of the procedure.

Increased work load in operating theatres, lack of awareness of sterilization and disinfection protocols, failure to properly dissemble, clean, and do high-level disinfection of laparoscope parts and improper storage continue to compromise patients' safety.

\section{Conclusion}

These three seemingly sporadic cases of post laparoscopic port site infections caused by nontuberculous mycobacteria over an 18 month period surfaced a breach in infection control standards in the operating theatres of a teaching hospital in Sri Lanka. The establishment of adequate infection control pertaining to laparoscopes with continuing education, visual inspection for surface cleaning; microbiological sampling and frequent audits are required to ensure compliance with good practice as use of laparoscopic surgical procedures increase. 


\section{Conflict of interest}

None of the authors have financial or non -financial conflicts of interest.

\section{Acknowledgments}

We would like to acknowledge the surgical and gynaecological teams at Colombo North Teaching Hospital, Respiratory Physicians at Central Chest Clinic and Hospital, Welisara and Consultant Microbiologist and the staff at National Tuberculosis Reference Laboratory, Welisara for their contribution on management of these patients.

\section{References}

1. Phillips MS, von Reyn CF. Nosocomial infections due to nontuberculous mycobacteria. Clin Infect Dis 2001; 33:1363-74. doi: http://dx.doi.org/10.1086/323126

2. Sasmal PK, Mishra TS, Rath S, et al. Port site infection in laparoscopic surgery: A review of its management. World J Clin Cases 2015; 3(10):864-871. doi: http://dx.doi.org/10.12998/wjcc.v3.i10.864

3. Chaudhuri S, Sarkar D, Mukerji R. Diagnosis and management of atypical mycobacterial infection after laparoscopic surgery. Indian J Surg 2010; 72(6):438-442. doi: http://dx.doi.org/10.1007/s12262-010-0164-7

4. Choudhury DK, Prakash R. Atypical mycobacterial infection following laparoscopic inguinal hernia repair: experience in five cases. IOSR J Dent Med Sci. 2016;15(2):40-42. doi: 10.9790/0853-152114042.

5. Sharma AK, Sharma R, Sharma S. Port site infection in laparoscopic surgeries. Ind Med Gaz. 2013; 224-229. No doi

6. Vijayaraghavan R, Chandrashekhar R, Sujatha Y,Belagavi CS. Hospital outbreak of atypical mycobacterial infection of port sites after laporoscopic surgery. J Hosp Infect 2006; 64(4)344-347 doi: http://dx.doi.org/10.1016/j.jhin.2006.07.021

7. Rutala WA, Weber DJ et al. Guideline for disinfection and sterilization in healthcare facilities. Centre for Disease Control and Prevention 2008 https://www.cdc.gov/infectioncontrol/guidelines/disinfection/cleaning.html 